\section{Summary \\ Evidence that oncogene activa- tion contributes to the induction and maintenance of human can- cers is becoming increasingly compelling. Specific mechanisms leading to neoplastic transforma- tion include point mutations, chromosomal translocations, gene amplification or deletion and other destabilizing genetic events. Animal studies have shown the high induction of tumors by chemical carcinogens, the ras oncogenes often being the target for DNA-damaging agents where activation is correlated with their known mutagenic effects. In man, one of the best examples of the relationship bet- ween unrepaired DNA lesions, mutations and carcinogenesis is the syndrome, Xeroderma pig- mentosum (XP). The absence of excision repair in this rare, auto- somal recessive disorder, results in a high predisposition to neo-

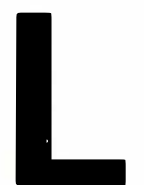
plasms in the sun-exposed skin. We have screened 8 XP tumors for activated transforming genes using $3 T 3$ transfection. In 2 tumors from a XP child, an activated $N$-ras oncogene was detected. Characterization by synthetic oligonucleotide probes showed a critical base substitution in codon $6 \mathrm{l}$, at a potential pyrimidine dimer. Many of the XP tumors showed $\mathrm{Ha}$-ras gene rearrangement and amplification sometimes together with c-myc amplification. The presence of several altered oncogenes in these $\mathrm{XP}$ tumors is probably due to the high level of unrepaired UV. induced lesions which persist in the skin. Hence XP serves as a very good model for studying carcinogenesis in man where specific lesions result in an initiating mutation (e.g. $N$-ras activation) which together with amplified gene (e.g. Ha-ras, c-myc) expression lead to tumor formation.

\title{
Activation des oncogènes dans les tumeurs épithéliales isolées de malades atteints du Xeroderma pigmentosum
}

\author{
Alain Sarasin, Leela Daya-Grosjean, \\ Horacio G. Suarez, Bertrand Chrétien, \\ Marie-Françoise Avril
}

'activation d'oncogènes est en partie liée à l'initiation de la cancérogenèse [1]. Quatre processus différents d'activation des oncogènes ont été décrits et analysés : l'insertion d'un rétrovirus près d'un proto-oncogène; la translocation chromosomique; l'amplification génique et la mutation ponctuelle. Dans 10 à $15 \%$ des tumeurs humaines, il a été détecté des mutations ponctuelles sur certains proto-oncogènes (essentiellement ceux de la famille ras) qui confèrent au gène muté la capacité de transformer des lignées établies de fibroblastes de souris (NIH 3T3) ou de rat [2]. Cette activité transformante est liée à une modification d'un acide aminé à un endroit précis de la protéine ras (P21) rendant cette protéine incapable d'effectuer son cycle enzymatique normal. Cette enzyme semble être impliquée dans la transduction de signaux à travers la membrane cytoplasmique [3].

\section{Le rôle majeur des lésions} sur l'ADN

Les cancérogènes chimiques induisent avec une forte fréquence l'apparition de cancers chez les animaux traités. L'étude de l'activation des gènes ras dans ces tumeurs chimioinduites a montré que la nature du changement de base était liée au type de cancérogène utilisé et au type de lésion induite sur la molécule d'ADN. Ainsi certaines substances alkylantes (cancérogènes chimiques, drogues antitumorales) ont la propriété de former des lésions $\mathrm{O}^{6}$. méthyl-guanine sur l'ADN traité. En l'absence d'une réparation parfaite de cette lésion, celle-ci pourra s'apparier au moment de la réplication de l'ADN lésé avec une thymine au lieu de la cytosine qui s'apparie naturellement avec la guanine non modifiée. Dans ces conditions, la conséquence de la présence de $\mathrm{l}^{1} \mathrm{O}^{6}-\mathrm{Me}-\mathrm{G}$ sera une mutation ponctuelle transformant une paire de bases $\mathrm{G}: \mathrm{C}$ en A:T. Ainsi, Barbacid a montré que dans $100 \%$ des cancers mammaires de rat soumis à l'alkylant nitrosométhylurée (NMU), l'activation du gène Ha-ras était due à une mutation ponctuelle de la paire de bases G:C en A:T correspondant au codon 12 de la protéine P2I (3). Le spectre des mutations obtenu sur les oncogènes activés provenant de tumeurs animales consécutives au traitement par des cancérogènes est donc similaire au spectre des mutations déterminé 
sur des systèmes modèles, in vitro, ou chez les bactéries après traitement de l'ADN par ces mêmes cancérogènes. Il serait particulièrement intéressant de pouvoir établir ce même type de corrélation chez l'homme et de pouvoir en déduire le mécanisme de la cancérogenèse à partir de l'étude du spectre de mutation observé sur les proto-oncogènes mutés. Le Xeroderma pigmentosum est, chez l'homme, l'exemple le plus pertinent où il apparaît possible d'établir ce type de corrélation. En effet, cette maladie transmise de façon héréditaire et récessive se caractérise par l'apparition, avec une fréquence particulièrement élevée, de tumeurs cutanées dans les zones exposées au soleil $[4,5]$. Cette cancérogenèse est liée à la présence de lésions induites par les UV sur l'ADN cellulaire (en particulier les dimères de pyrimidine et les pyrimidines (6-4) pyrimidones). L'absence de réparation de l'ADN lésé, caractéristique des cellules isolées de malades atteints du Xeroderma pigmentosum, est directement responsable de la production de mutations ponctuelles localisées en face de la lésion, c'est-à-dire de deux pyrimidines adjacentes (figure 1).

\section{Les lésions sur l'ADN sont-elles responsables de l'activation des oncogènes?}

Nous avons analysé l'ADN provenant de huit tumeurs épithéliales (carcinomes baso- ou spinocellulaires), prélevées chirurgicalement chez des enfants XP, pour leur activité transformante dans le test des cellules de souris NIH 3T3. Les $\mathrm{ADN}$ isolés de deux tumeurs indépendantes provenant du même malade (BY) ont été capables d'induire des foyers de cellules transformées. L'activité transformante était due au gène $N$-ras (figure $2 A$ ) $[4,6]$. Nous avons déterminé la mutation ponctuelle responsable de cette activité transformante, en utilisant la méthode d'amplification génique in vitro suivie d'hybridation différentielle avec des oligonucléotides synthétiques (figure 2B) [7]. Le codon 61 $\mathrm{du}$ gène $N$-ras transformant a subi une mutation ponctuelle ce qui le fait coder pour l'histidine au lieu de la glutamine [8] (figure 3). Cette modification est suffisante pour

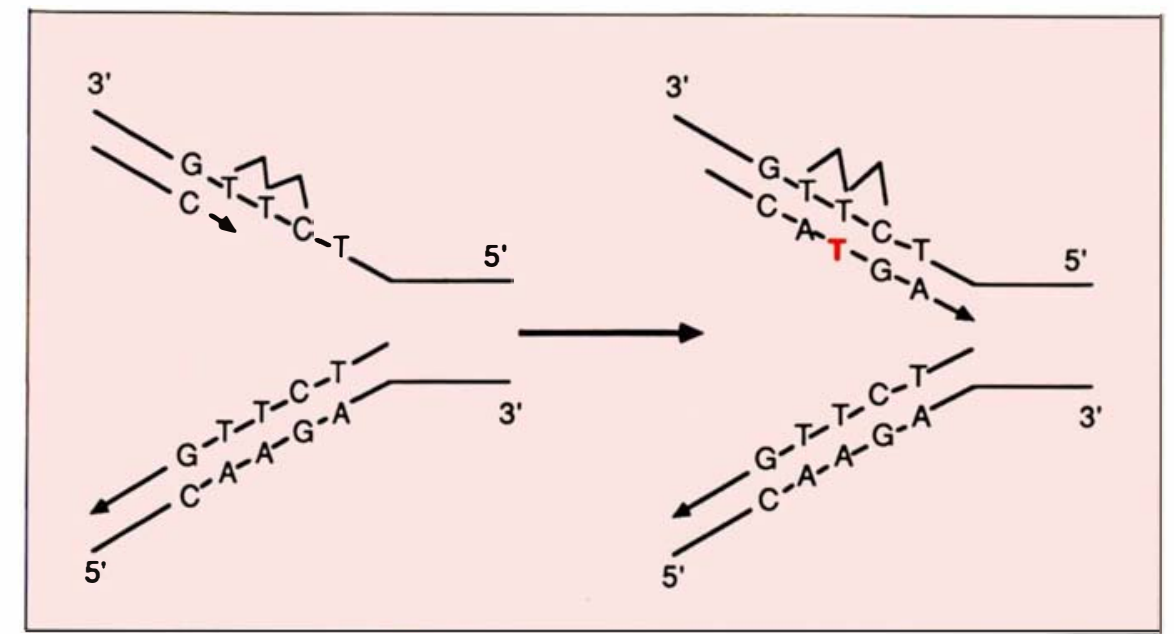

Figure 1. Modèle de création de mutation ponctuelle pendant la réplication de I'ADN contenant des lésions induites par les UV. La fourche de réplication est normalement bloquée par les lésions produites par les UV entre deux thymines adjacentes: dimère de thymine ou thymine (6-4) thymine [4]. Une éventuelle réplication de ces lésions ( $\overparen{T}$ ou $\widehat{T C}$ ) se traduira par une erreur d'appariement conduisant à une mutation ponctuelle opposée à l'une des bases impliquées dans la lésion. Les séquences indiquées correspondent à celles entourant le codon 61 du gène $\mathrm{N}$-ras (voir figure 3 ).

conférer au gène $N$-ras ainsi muté une activité de transformation cellulaire [3].

Nous pouvons donc faire l'hypothèse qu'après irradiation UV des cellules épithéliales de la peau exposée, et en absence de réparation de l'ADN, une lésion s'est produite sur les pyrimidines adjacentes en position 182-183 ou 183-184 du gène $N$-ras. La réplication d'une telle lésion peut alors donner naissance à une erreur d'appariement conduisant à l'incorporation de la thymine, au lieu de l'adénine, en face d'une des thymines impliquées dans la lésion (figures 1 et 3). Ce type de mutation est observé fréquemment avec des systèmes modèles in vitro ou des sondes exogènes in vivo telles que les virus navettes ou les virus animaux (SV40) irradiés aux UV [9, 10]. Cette activation du gène $N$-ras n'existe pas dans les fibroblastes non transformés du même malade. Ceci indique que la prédisposition génétique évidente à développer des cancers chez le malade XP n'est pas due à une transmission héréditaire d'un gène $N$-ras muté.

De façon intéressante, Van der Lubbe et al. [11] ont irradié aux UV in vitro l'ADN cloné du proto-oncogène $N$ ras et testé directement son activité transformante après transfection de l'ADN lésé dans des cellules de souris NIH-3T3. La grande majorité des mutations ponctuelles se trouvaient sur le codon 61 du gène $N$-ras au niveau d'une des deux thymines de ce codon. Ainsi la même conclusion que celle déduite des résultats sur les tumeurs XP a été proposée, à savoir l'induction d'une lésion sur les pyrimidines 182-183-184 suivie de mutations au niveau de l'une des thymines du codon 6l. De plus, le traitement in vitro de l'ADN irradié par une enzyme spécifique, qui élimine les dimères de pyrimidine et non les $\mathrm{Py}(6-4) \mathrm{Py}$, diminue fortement l'activité transformante de l'ADN. Ceci suggère que les dimères de pyrimidine sont essentiellement responsables de cette mutagenèse [ll].

Dans l'exemple humain du XP, nous aurions donc une situation comparable à celle obtenue lors de traitements par des cancérogènes donnés chez les animaux. Nous pouvons donc en déduire un mécanisme précis d'activation des oncogènes, par mutation 
ponctuelle, à partir d'un type donné de lésions sur l'ADN cellulaire.

\section{Amplification d'oncogènes sous l'effet des ultraviolets}

Il est toutefois connu que l'activation par mutation ponctuelle d'un gène ras n'est pas suffisante pour permettre le développement tumoral [3]. Ainsi, il a été démontré que des tumeurs bénignes de la peau (papillomes) d'animaux traités par des cancérogènes faibles contiennent déjà un gène ras (souvent Ha-ras 1) transformant, bien que la grande majorité des ces tumeurs régressent et ne donnent jamais de cancer [12]. Il semble que des événements génétiques secondaires soient nécessaires pour promouvoir le processus tumoral à partir d'une cellule initiée. Dans cette optique, nous avons recherché dans les tumeurs épithéliales de malades XP s'il existait d'autres modifications génétiques, en particulier au niveau des autres oncogènes. Ainsi, nous avons démontré l'existence d'amplification de proto-oncogènes ( $c$-myc et/ou Ha-

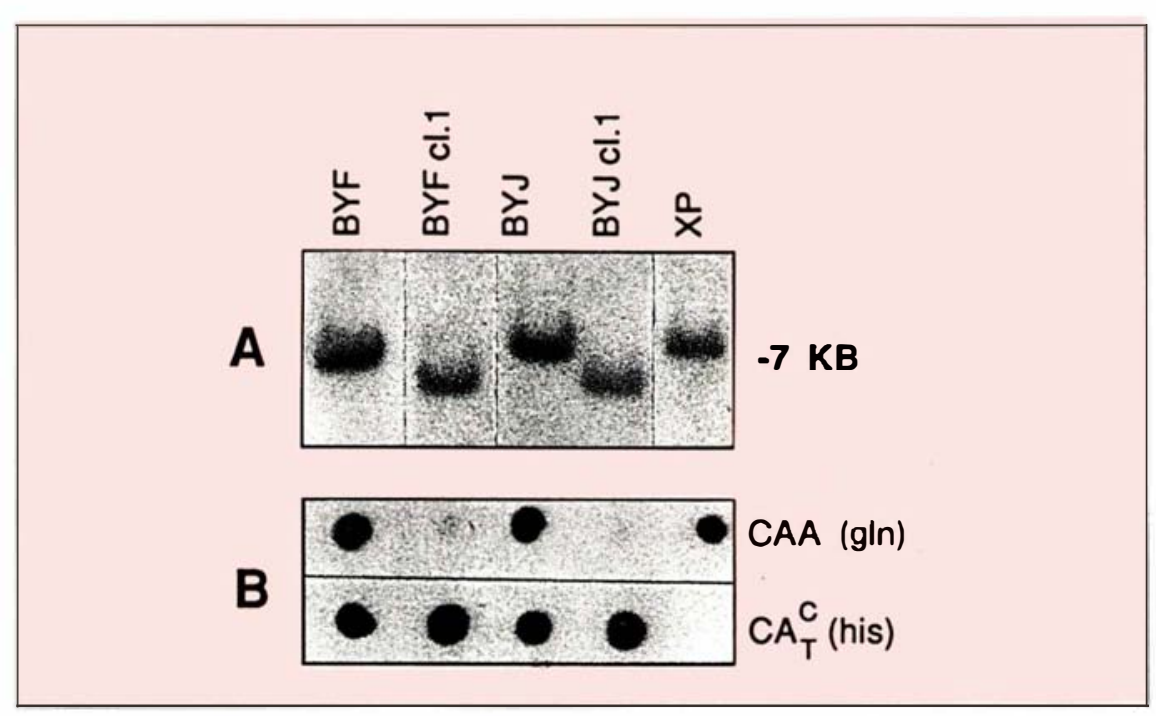

Figure 2. Activation du gène N-ras dans les tumeurs XP. A. L'ADN isolé de tumeurs épithéliales XP est transfecté dans les cellules de souris NIH 3T3. La présence d'un oncogène activé est détectée par l'apparition de foyers cellulaires (transfectants primaires) correspondant à des cellules transformées par l'oncogène. $L A D N$ de deux tumeurs XP (BYF et BYJ), de deux transfectants primaires (BYF cl. 1 et $B Y J$ cl. 1) et de fibroblastes normaux, non transformés de XP (XP) est analysé pour la présence de l'oncogène $\mathrm{N}$-ras par la technique de Southern. $20 \mu \mathrm{g}$ d'ADN de haut poids moléculaire sont digérés par EcoRl, séparés par électrophorèse sur gel d'agarose $1 \%$ et hybridés à différentes sondes d'oncogènes marquées au ${ }^{32} P$ afin de déterminer lequel est transféré et donc activé dans les tumeurs primitives. L'oncogène $\mathrm{N}$-ras humain est le seul oncogène détecté dans les cellules de souris transformées, indiquant ainsi qu'il est responsable de la transformation cellulaire.

B. Hybridation différentielle d'ADN avec des oligonucléotides synthétiques spécifiques du codon 61 du gène $N$-ras normal (CAA) ou muté (CAC/CAT) (voir figure 3). Les ADN génomiques sont amplifiés par la réaction en chaîne de polymérase (30 cycles) (PCR, voir $\mathrm{m} / \mathrm{s} n^{\circ} 8, \mathrm{vol} .4$, p. 515), déposés sur filtre de nitrocellulose, puis hybridés avec des oligonucléotides (20 mères) marqués au 32P [7, 8]. Ces résultats montrent que les cellules transformées de souris contiennent un gène humain $\mathrm{N}$-ras avec une mutation $(A \rightarrow C$ ou $A \rightarrow T)$ sur le codon 61. L'utilisation d'oligonucléotides synthétiques purs complémentaires du codon CAC ou CAT a montré que la mutation ponctuelle est en fait une transversion $A \rightarrow T$. Les tumeurs $X P$ intiales (BYF et BYJ) contiennent un allèle normal du gène $\mathrm{N}$-ras (CAA) et un allèle muté du gène $\mathrm{N}$-ras activé (CAC/CAT). gln = glutamine; his = histidine.

$\mathrm{m} / \mathrm{s} n^{\circ} 10$ vol. 4 , décembre 88 ras) dans un nombre important de tumeurs [6, 8]. En particulier, le gène Ha-ras est amplifié et quelquefois réarrangé dans $30-40 \%$ des tumeurs épithéliales XP (figure 4), ce qui est significativement différent de ce qui se passe en général dans l'ensemble des tumeurs humaines, où ce chiffre est estimé autour de $1 \%$ [3]. Il est donc intéressant de déterminer s'il existe un rapport entre le fort taux d'amplification génique observé dans les tumeurs des malades XP et le type de lésions responsables de la mutagenèse. En effet, il a été démontré que les lésions qui bloquent la progression normale de la réplication de l'ADN, induisent l'amplification non spécifique de portions d'ADN, probablement grâce à des cycles multiples de réinitiation [13]. Les dimères de pyrimidine sont particulièrement aptes à induire de tels cycles de réplication abortive. Ainsi, à nouveau la connaissance de l'agent initiateur des tumeurs (les lésions UV-induites) nous permet d'envisager un scénario de tumorigenèse chez l'homme. La présence de lésions non réparées sur l'ADN cellulaire va conduire au cours de la réplication au blocage de la synthèse d'ADN, suivi de cycle de réinitiation et donc à l'amplification de séquences probablement au hasard. Si un gène conférant un avantage sélectif $(c-m y c$ ou Ha-ras) est amplifié, entraînant ainsi la surexpression de la protéine correspondante, la cellule proliférera plus rapidement et pourra se transformer en cellule tumorale à condition qu'un second oncogène (par ex. $N$-ras activé) ait été préalablement muté suite à l'action de ces mêmes lésions UV-induites.

\section{Conclusion}

Nos résultats concernant le Xeroderma pigmentosum suggèrent que les hypothèses effectuées à partir des expériences de cancérogenèse chez l'animal traité par des agents mutagènes bien déterminés sont applicables à l'homme. Ainsi, lorsque l'on connaît de façon précise l'agent causal du développement tumoral, il est possible de corréler le type de lésions primaires sur l'ADN à la nature des modifications génétiques impliquées dans la cancérogenèse. Il est évident que dans la plupart des cancers hu- 


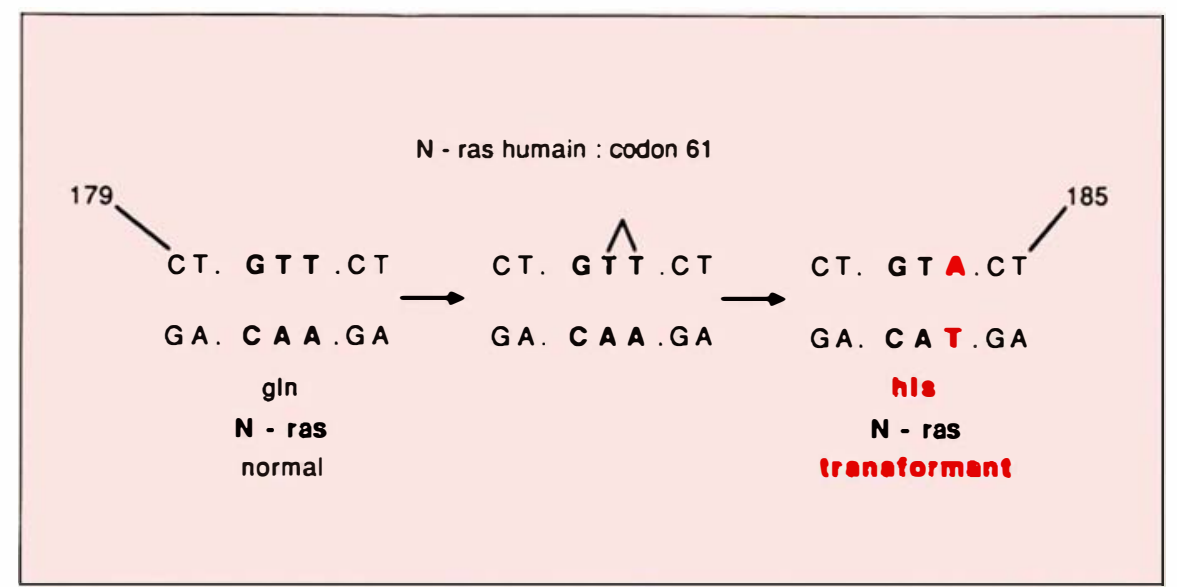

Figure 3. Modèle de l'activation du proto-oncogène N-ras par mutation ponctuelle. L'irradiation UV induit des lésions sur deux thymines adjacentes (TT) du codon 61. En absence de réparation de I'ADN, les cellules XP produisent une mutation ponctuelle en face de cette lésion (figure 1) conduisant à une modification du code génétique. Les chiffres correspondent à la numérotation des nucléotides de la partie codante du gène $\mathrm{N}$-ras [14]. mains, nous ne connaissons pas l'origine des modifications génétiques délétères. On peut envisager la présence de lésions spécifiques provenant de cancérogènes chimiques ou physiques (hydrocarbures polycycliques, nitrosamines, amiante, agents alkylants, mycotoxines ou rayonnements ionisants), de lésions cryptiques provenant de modifications spontanées de certaines bases nucléiques (déamination des cytosines ou des méthyl-cytosines) ou d'erreurs au cours de la réplication ou de la réparation de l'ADN. Ces modifications auront probablement un spectre d'action différent les uns des autres, ce qui pourrait expliquer que si l'on prend l'ensemble des cancers «spontanés » chez l'homme, il n'existe pas de modification consensuelle des proto-oncogènes en fonction du type du cancer ou de sa localisation anatomique. Une meilleure connaissance à la fois des lésions initiatrices des cancers et des mécanismes conduisant à l'activation des proto-oncogènes est nécessaire pour améliorer la prévention et le traitement des tumeurs

\section{RÉFÉRENCES}

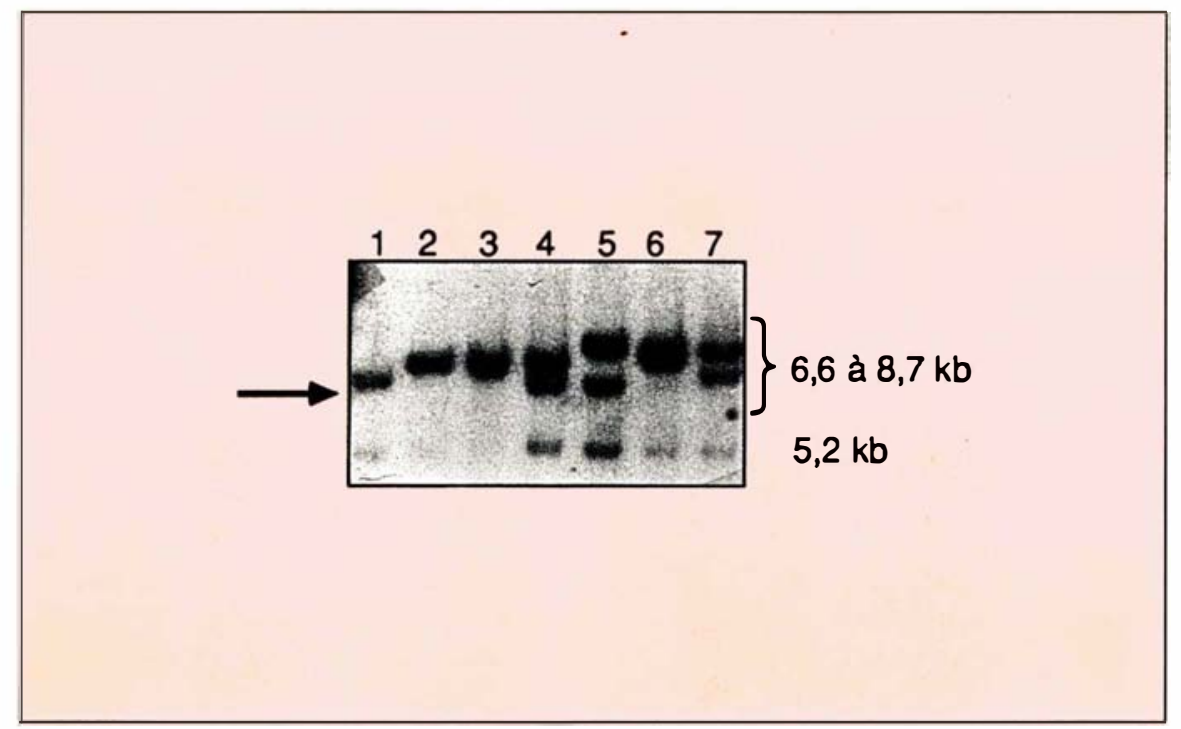

Figure 4. Amplification du gène Ha-ras dans les tumeurs XP. Les ADNs génomiques provenant de tumeurs épithéliales XP ont été analysés après digestion avec l'enzyme Bam H1, par électrophorèse dans un gel d'agarose $1 \%$, transférés sur feuille de nitrocellulose et hybridés avec la sonde spécifique du gène Ha-ras ( $D E J)$, marquée au ${ }^{32} P$. Voie 1 : fibroblastes normaux XP; voies 2-7: six tumeurs $X P$ provenant de malades différents. L'amplification et éventuellement le réarrangement du fragment normal $(6,6 \mathrm{~kb}$ : flèche) du gène Ha-ras sont évidents dans les tumeurs $X P$.
1. Stehelin D. Les oncogènes cellulaires, clés de la cancérogenèse. médecine/sciences 1985 ; $1: 12-6$.

2. Loucheux-Lefebvre MH. Activation des oncogènes par mutation ponctuelle. médecine/sciences $1987 ; 3$ : 33-7.

3. Barbacid M. Ras genes. Ann Rev Biochem $1987 ; 56: 779-827$.

4. Sarasin A, Renault G, Blanchet-Bardon C, Boue J, Dumez Y. Le Xeroderma pigmentosum : caractéristiques cliniques, génétiques et cellulaires. Développement d'un test anténatal. médecine/sciences 1988 ; 4 : 608-17.

5. Hanawalt PC, Sarasin A. Cancer prone hereditary diseases with DNA processing abnormalities. Trends Genet 1986 ; 2 : 124-9.

6. Suarez HG, Nardeux PC, Andeol Y, Sara$\sin$ A. Multiple activated oncogenes in human tumors. Oncogene Res 1987; 1 : 201 -

7. Bos JL, Verlaan-de-Vries M, Marshall CJ, Veeneman GH, Van Boom JH, Van der Eb AJ. A human gastric carcinoma contains a single mutated and an amplified normal allele of the Ki-ras oncogene. Nucleic Acids Res 1986 ; 14: 1209-17.

$\mathrm{m} / \mathrm{s} n^{\circ} 10 \mathrm{vol} .4$, décembre 88 
8. Suarez HG, Daya-Grosjean L, Schlaifer D, et al. Activated oncongenes in skin tumors from a repair deficient syndrome, Xeroderma pigmentosum. Cancer Res 1988 (sous presse).

9. Bourre F, Sarasin A. Targeted mutagenesis of SV40 DNA induced by UV light. Nature $1983 ; 305: 68-70$

10. Bredberg A, Kraemer KH, Seidman MM. Restricted ultraviolet mutational spectrum in a shuttle vector propagated in Xeroderma pigmentosum cells. Proc Natl Acad Sci USA $1986 ; 83: 8273-7$.

11. Van der Lubbe JLM, Rosdorff H JM Bos JL, et al. Activation of $N$-ras by ultraviolet irradiation in vitro. Oncogene Res 1988 (sous presse).

12. Balmain A, Ramsden M, Bowden GT, et al. Activation of the mouse cellular Harveyras gene in chemically induced benign skin papillomas. Nature $1984 ; 307$ : 658-60.

13. Lavi S. Carcinogen-mediated amplification of viral DNA sequences in simian virus 40-transformed Chinese hamster embryo cells. Proc Natl Acad Sci USA 1981; 78 : 61 44-8.

14. Taparowsky E, Shimizu K, Goldfarb M, et al. Structure and activation of the human $N$-ras gene. Cell $1983 ; 34: 581-6$.

\section{Remerciements}

Nous tenons à remercier C. Drougard, P. Nardeux et J.-A. Duvillard pour leur excellente aide technique. Nous sommes particulièrement reconnaissant aux Drs A. Margulis, Y. Decroix, J.-P. Cesarini, M. Vuillaume et J. Coppey pour l'obtention de pièces opératoires. Ce travail a été possible grâce à l'aide de l'ARC, la LFNCC, le GEFLUC et la CEE (contrat $\mathrm{n}^{\circ} \mathrm{B10}-\mathrm{I63F}$ ).

\section{ADRESSES}

A. Sarasin: directeur de recherche au Cnrs. L. Daya-Grosjean: chargée de recherche au Cnrs. H.G. Suarez: directeur de recherche au Cnrs. Laboratoire de génétique moléculaire, Institut de recherches scientifiques sur le cancer, BP $n^{\circ} 8,94802$ Ville juif Cedex, France. B. Chrétien: chef de clinique. Clinique chirurgicale infantile, hôpital Necker Enfants-Malades, 149, rue de Sèvres, 75015 Paris, France.

M.-F. Avril : chef de service. Service de dermatologie, Institut Gustave-Roussy, rue Camille-Desmoulins, 94805 Villejuif, France.

\section{TIRÉS A PART}

A. Sarasin. 